\title{
Efficacy and safety of prolonged-release melatonin for insomnia in middle-aged and elderly patients with hypertension: a combined analysis of controlled clinical trials
}

\author{
This article was published in the following Dove Press journal: \\ Integrated Blood Pressure Control \\ 24 January 2012 \\ Number of times this article has been viewed
}

\author{
Patrick Lemoine' \\ Alan G Wade ${ }^{2}$ \\ Amnon Katz ${ }^{3}$ \\ Tali $\mathrm{Nir}^{3}$ \\ Nava Zisapel ${ }^{3,4}$ \\ 'The Clinique Lyon-Lumière, Meyzieu, \\ France; ${ }^{2}$ CPS Research, 3 Todd \\ Campus, Glasgow, UK; ${ }^{3}$ Neurim \\ Pharmaceuticals Ltd, Tel-Aviv, Israel; \\ ${ }^{4}$ Department of Neurobiology Faculty \\ of Life Sciences, Tel-Aviv University, \\ Tel-Aviv, Israel
}

\begin{abstract}
Background: Add-on prolonged-release melatonin (PRM) in antihypertensive therapy has been shown to ameliorate nocturnal hypertension. Hypertension is a major comorbidity among insomnia patients. The efficacy and safety of PRM for primary insomnia in patients aged 55 years and older who are treated with antihypertensive drugs were evaluated.

Methods: Post hoc analysis of pooled antihypertensive drug-treated subpopulations from four randomized, double-blind trials of PRM and placebo for 3 weeks $(\mathrm{N}[\mathrm{PRM}]=195$; $\mathrm{N}[$ placebo $]=197)$ or 28 weeks $(\mathrm{N}[\mathrm{PRM}]=157$; $\mathrm{N}[$ placebo $]=40)$. Efficacy measurements included Leeds Sleep Evaluation Questionnaire scores of quality of sleep and alertness and behavioral integrity the following morning after 3 weeks, and sleep latency (daily sleep diary) and Clinical Global Impression of Improvement (CGI-I) after 6 months of treatment. Safety measures included antihypertensive drug-treated subpopulations from these four and three additional single-blind and open-label PRM studies of up to 1 year $(\mathrm{N}[\mathrm{PRM}]=650$; $\mathrm{N}$ [placebo] $=632$ ).
\end{abstract}

Results: Quality of sleep and behavior following wakening improved significantly with PRM compared with placebo $(P<0.0001$ and $P<0.0008$, respectively). Sleep latency $(P=0.02)$ and CGI-I $(P=0.0003)$ also improved significantly. No differences were observed between PRM and placebo groups in vital signs, including daytime blood pressure at baseline and treatment phases. The rate of adverse events normalized per 100 patient-weeks was lower for PRM (3.66) than for placebo (8.53).

Conclusions: The findings demonstrate substantive and sustained efficacy of PRM in primary insomnia patients treated with antihypertensive drugs. PRM appears to be safe for insomnia in patients with cardiovascular comorbidity.

Keywords: prolonged-release melatonin, hypertension, nocturnal blood pressure, insomnia, cardiovascular disease, sleep quality

\section{Introduction}

Insomnia, defined as difficulties initiating or maintaining sleep or nonrestorative sleep associated with significant daytime distress (Diagnostic and Statistical Manual of Mental Disorders, 4th ed [DSM-IV]), occurs in about 30\% of subjects aged 55 years and older. ${ }^{1-6}$ One of the major health issues found in the $55+$ years population is hypertension. ${ }^{7,8}$ The prevalence of hypertension is significantly higher among insomnia patients $(\sim 44 \%)$ as compared with good sleepers $(\sim 19 \%)$, suggesting a cross-talk between sleep and blood pressure (BP) control. ${ }^{9}$ In particular, higher systolic BP and lower day-to-night systolic BP dipping were reported in normotensive insomniacs as
Correspondence: Amnon Katz

Neurim Pharmaceuticals Ltd,

HaBarzel 27 St Tel-Aviv, Israel

Tel +97237684969

Fax +97236494568

Email amnonk@neurim.com 
compared with in normotensive good sleepers. ${ }^{10}$ Furthermore, short sleep duration and insomnia were found to be risk factors for hypertension, as assessed in middle-aged subjects and depressed patients. ${ }^{11,12}$ In the elderly, it was shown that impaired sleep architecture as expressed by decreased slowwave sleep increases the risk of developing hypertension. ${ }^{13}$ The blunted nocturnal BP dip and the resulting nocturnal hypertension have severe consequences and are considered major risk factors for cardiovascular events. ${ }^{14}$ Accordingly, a recent Dutch population-based cohort study of 20,432 men and women aged 20-65 years revealed that short sleepers with poor sleep quality had a $63 \%$ higher risk of cardiovascular disease (CVD) and a 79\% higher risk of coronary heart disease compared with normal sleepers with good quality sleep. ${ }^{15}$ Add-on controlled-release and prolonged-release melatonin (PRM) preparations for antihypertensive therapy have been shown to ameliorate nocturnal hypertension. ${ }^{16}$ It is therefore important to find out whether such preparations would effectively treat insomnia in patients who have cardiovascular comorbidity.

PRM (Circadin ${ }^{\circledR}$, Rad Neurim Pharmaceuticals EEC Ltd, Reading, UK) is a new drug licensed to treat primary insomnia in patients aged 55 years and older. It is designed to mimic the release pattern of endogenous melatonin, a hormone that regulates sleep and circadian rhythms. ${ }^{17}$ There is an age-related decline in the robustness of the biological clock and melatonin production, thus depriving the brain of an important sleep regulator. ${ }^{18-21}$ In patients aged 55 years and over who suffer from poor sleep quality, melatonin production is even lower than in healthy elderly without such a complaint. ${ }^{22,23}$ PRM (2 mg) has been shown to be effective in improving the patient-reported quality of sleep and morning alertness as well as sleep latency in insomnia patients. ${ }^{24-28}$ It was thus pertinent to check whether add-on of PRM improves quality of sleep, sleep latency, and next-day alertness in patients aged 55 and older with primary insomnia who are treated with antihypertensive drugs. The safety of PRM in this population was also of interest because of potential drug interactions with medications used for the treatment of CVD, including hypertension.

The efficacy and safety of treatment with PRM and placebo were thus compared in a subpopulation from PRM clinical trials of the insomnia patients aged 55 years and older treated with antihypertensive drugs when entering the studies. A post hoc, pooled analysis of four randomized, double-blind trials (short-term 3-week studies and a long-term 6-month study $)^{24-28}$ with similar designs compared the efficacy of PRM and placebo ( 3 weeks up to 6 months) in the treatment of primary insomnia in this subpopulation. Safety measures included vital signs as well as the frequencies of adverse events (AEs) along with general safety measures in all patients with insomnia from these four and three additional single-blind and open-label PRM trials that had a recorded history of any cardiovascular abnormalities when entering the trials.

\section{Methods}

\section{Studies: efficacy analysis}

Data from four clinical trials conducted between 1998 and 2008 were used for the efficacy analysis. ${ }^{24-28}$ These trials shared the same basic design, which included 1-2 weeks of a single-blind placebo run-in period followed by a 3-week randomized, double-blind treatment period. Patients were instructed to take either PRM $2 \mathrm{mg}\left(\right.$ Circadin $\left.^{\circledR}\right)$ or a placebo tablet daily 2 hours before bedtime. Efficacy parameters were measured at the beginning (baseline) and at the end of the 3 -week double-blind period. In one of these studies, ${ }^{27}$ the double-blind treatment was then continued for 6 months, wherein patients randomized to PRM continued and those randomized to placebo were re-randomized to PRM and placebo for 6 months (Figure 1). Another study ${ }^{24}$ included a 6- to 12-month open-label extension phase of PRM $2 \mathrm{mg}$ treatment, which contributed safety data.

\section{Studies: safety analysis}

Safety analysis was performed for all patients included in the efficacy analysis. For the sake of completeness of the data, the analysis included also all patients with insomnia and any recorded CVD aged 55 years and older from two additional single-blind safety trials ${ }^{23,26}$ and a long-term openlabel extension phase of one study. ${ }^{24}$ In these trials, patients aged 18-80 years participated, and PRM doses used were $0.1 \mathrm{mg}, 1 \mathrm{mg}, 2 \mathrm{mg}$, and $5 \mathrm{mg}$ daily for 3-52 weeks. Altogether, the number of patients with any recorded CVD included in the safety analysis was $\mathrm{N}[\mathrm{PRM}]=650$ and $\mathrm{N}[$ placebo $]=632$.

All study protocols were approved by local ethics committees and complied with Good Clinical Practice standards stated in the Declaration of Helsinki 1975.

\section{Subjects}

In the included double-blind PRM trials, eligible patients were men and women aged 55-80 years suffering from primary insomnia according to DSM-IV criteria and for whom this was the main consultation complaint. A four-step process was used for screening out patients with secondary 

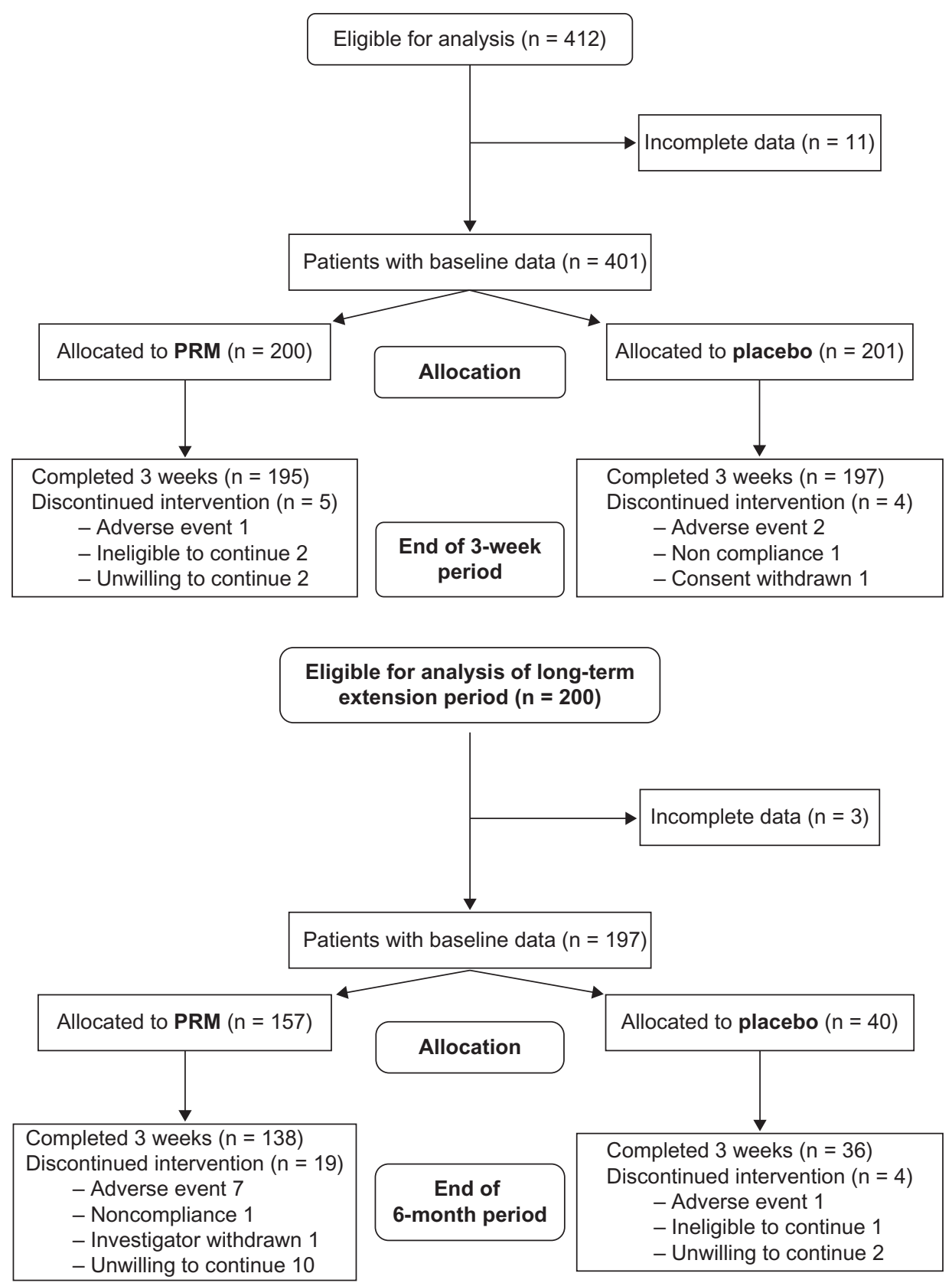

Figure I Overall patient disposition in efficacy analysis. Analysis of the short-term period included eligible patients who completed 3 weeks of double-blind treatment with prolonged-release melatonin (PRM) or placebo. Analysis of the long-term period included patients of one study ${ }^{27}$ re-randomized to PRM and placebo for 26 weeks of treatment.

sleep disorders, including depression and other sleep disorders. First, a Sleep History Questionnaire (SHQ) was used. This SHQ, which was adopted from the Management of Insomnia Guidelines for Clinical Practice of the World Psychiatric Association, characterizes the primary sleep complaint and also helps in differentiating primary insomnia from secondary insomnia due to medical and psychiatric disorders (including depression and anxiety) and specific insomnia disorders like circadian rhythm disorders, movement disorders, parasomnias, and breathing-related sleep disorders. Second, the SHQ was performed at the screening visit by a qualified clinician. Third, in order to rule out psychiatric disorders, including depression anxiety and dementia, the patients went through a detailed psychological assessment that included the Raskin Depression Scale, Covi Anxiety Scale, and Mini-mental State Examination at Visit 1. Finally, patients who were using psychotropic treatments (neuroleptics, antiepileptics, barbiturates, antidepressants, anxiolytics, and lithium) in the previous 3 months before the study were excluded. A positive drug screen on Visit 2 for 
benzodiazepines, barbiturates, sedating antihistamines, hydroxyzine, doxylamine, zaleplon, zopiclone, or zolpidem led to immediate exclusion. In addition, patients had to report any concomitant medication, and any patient who reported using any psychotropic treatment as detailed was not randomized in the study. For the purpose of the current analysis, patients were selected if their medical history included hypertension and if they received antihypertensive medication before and during the study.

\section{Efficacy measures}

The primary efficacy measures in the short-term (3 weeks) studies were the improvements in quality of sleep and morning alertness as assessed by the Leeds Sleep Evaluation Questionnaire (LSEQ). The LSEQ is a widely used standardized instrument for the measurement of sleep difficulties in clinical settings. ${ }^{29}$ It is a retrospective instrument by which the patients are asked to contrast aspects of their current sleep with those at the time before they joined the study. The LSEQ comprises ten individual visual analog scales (100 millimeters) shown by factor analysis to assess four discrete domains that are used independently to assess the following aspects of sleep and daytime behavior: getting to sleep, quality of sleep (QOS), awakening from sleep, and behavior following wakening (BFW). ${ }^{30,31}$ The QOS domain is the mean of Questions 4 and 5, which relate to the question "How would you describe the quality of your sleep compared with normal sleep?". Alertness and behavioral integrity the following morning (BFW) is the mean of Questions 8, 9, and 10 ("How do you feel when you wake up?, How do you feel now?, How would you describe your balance and coordination upon awakening?"). The LSEQ is used in a repetitive manner, yielding a series of measurements, and the difference between current and preceding measurements is used in drug efficacy evaluations..$^{29}$ Patients were asked to fill in the LSEQ 2 hours after awakening and to evaluate their quality of sleep and morning behaviors as compared with the respective values before starting run-in. Patients in all four studies completed the LSEQ during the last 3 days of the run-in period (baseline measurement) and the last 3 days of the 3-week treatment period. The changes in each parameter averaged over three consecutive days from run-in placebo (baseline) to end of the 3-week treatment were calculated for each patient. In the long-term study, ${ }^{27}$ patients completed a daily sleep diary. The main efficacy parameter in this study was the patient-reported time taken to fall asleep (sleep latency) measured over the last 7 days of baseline and treatment period. The global improvement in patients' health status, assessed in each patient using Clinical Global Impression of Improvement (CGI-I), ${ }^{32}$ is also presented as a measure of overall benefit to the patients. The CGI rating scales are commonly used measures of symptom severity, treatment response, and efficacy of treatments. This is a validated subjective scale that requires the user of the scale to compare the subjects with typical patients in the clinician experience. The CGI-I is a seven-point scale that requires the clinician to assess how much the patient's illness has improved or worsened relative to a baseline state at the beginning of the intervention and can be rated as 1 , very much improved; 2 , much improved; 3 , minimally improved; 4 , no change; 5 , minimally worse; 6 , much worse; or 7 , very much worse.

\section{Safety}

Vital signs, including BP (daytime), were measured at each visit as a measure of safety and tolerability. AE incidences were pooled, including those in patients for whom cardiovascular abnormality was recorded in either their medical history or the pretreatment physical examination. In an attempt to adjust for differences in duration of exposure between short- and long-term treatment periods with PRM and placebo, the incidences of AEs normalized for the exposure period (per 100 patient-weeks) were also presented.

\section{Statistical analysis}

Analyzed variables were presented in summary tables providing sample size $(\mathrm{N})$, arithmetic mean, standard deviation, and minimum and maximum values. An analysis of covariance (ANCOVA) model was applied for testing the significance of the differences in efficacy measures between the study groups adjusted to baseline value and study. $\chi^{2}$ analysis was applied for comparing responder rates assessed by the CGI-I score between PRM and placebo groups after 6 months of treatment. A responder was defined as a patient who scored 1 (very much improved) or 2 (improved) in CGI-I at the end of the 6-month period. Student's $t$-test analysis was applied for comparing mean group BP (daytime measurement) at baseline and after 3 weeks (for all studies) and 6 months (longterm study). ${ }^{27,28}$ Effect size was calculated using Cohen's $d .^{33}$ All tests applied were two-tailed, and a $P$ value $\leq 5 \%$ was considered statistically significant. The data were analyzed using $\mathrm{SAS}^{\circledR}$ (v 9.1; SAS Institute, Cary, NC).

\section{Results \\ Efficacy}

A total of 412 primary insomnia patients (139 men, 273 women) who had a medical history of hypertension and 
Table I Improvement in quality of sleep with PRM compared to placebo (3 weeks)

\begin{tabular}{|c|c|c|c|c|c|c|}
\hline & \multicolumn{3}{|c|}{ PRM } & \multicolumn{3}{|c|}{ Placebo } \\
\hline & $\mathbf{N}$ & Mean (millimeters) & SD & $\mathbf{N}$ & Mean (millimeters) & SD \\
\hline Baseline & 200 & 52.8 & 14.5 & 201 & 53.4 & 13.9 \\
\hline 3 weeks & 195 & 43.9 & 14.4 & 197 & 49.5 & 14.5 \\
\hline Mean change from baseline & 195 & -9.2 & 16.2 & 197 & -3.7 & 15.0 \\
\hline Significance PRM vs placebo & \multicolumn{6}{|c|}{$P<0.0001$} \\
\hline
\end{tabular}

Abbreviations: PRM, prolonged-release melatonin; SD, standard deviation.

concomitant therapy records of hypertension (with or without other CVD) were included in the PRM trials. Of these, 392 completed the 3 -week randomized, double-blind treatment period, had valid efficacy data, and were included in the analysis (see Figure 1 for full details). All of these subjects were concomitantly treated with at least one antihypertensive drug (26\% with ß-blockers, 53\% with renin-angiotensin system inhibitors, and 33\% with calcium channel blockers). The majority of patients received two or more kinds of antihypertensive medications concomitantly, and the minority $(19.6 \% ; \mathrm{N}=77)$ received one kind of antihypertensive medication (3.8\% ß-blockers, $6.1 \%$ angiotensin-converting enzyme inhibitors, $3.6 \%$ calcium channel inhibitors, and $6.1 \%$ other kind of medication such as diuretics and serum lipidreducing agents).

The effects of PRM (Circadin ${ }^{\circledR}, 2 \mathrm{mg}$ ) and placebo treatment on sleep quality in this population are presented in Table 1 . The patients have demonstrated a statistically significant improvement in QOS with PRM for 3 weeks compared with placebo with a mean improvement (decrease) from baseline of 9.2 with PRM versus 3.7 millimeters with placebo $(\mathrm{df}=1 ; F=16.67$; $P<0.0001$, ANCOVA adjusted to baseline and study). The effect of PRM on BFW the following morning is presented in Table 2. A statistically significant improvement in BFW with PRM for 3 weeks compared with placebo was found, with a mean improvement (decrease) from baseline of 7.2 with PRM versus 3.0 millimeters with placebo $(\mathrm{df}=1 ; F=11.9 ; P<0.0008$, ANCOVA adjusted to baseline and study). The corresponding effect size (Cohen's $d$ ) was 0.35 for QOS and 0.3 for BFW.

By the end of the 6-month treatment period, the mean improvement (decrease) in patients' evaluated sleep latency (reported in the daily sleep diary) was significantly higher with PRM (25.89 minutes) than with placebo (7.54 minutes) (df $=1 ; F=8.74 ; P=0.02$, ANCOVA) (Table 3$)$. The Cohen's $d$ effect size was 0.39 .

Following the 6-month treatment period, $38.9 \%$ of the patients improved or very much improved in CGI-I as compared with $12 \%$ of placebo-treated patients $\left(\chi^{2}=7.87\right.$; $P=0.0003)$.

The association between the concomitant antihypertensive therapy and response to PRM could not be obtained, because only $\sim 20 \%$ of the patients included in the analysis had been treated with one kind of antihypertensive medication, and the vast majority $(\sim 80 \%)$ of the patients had been treated with more than one antihypertensive medication concomitantly. For about $26 \%$ of patients the antihypertensive medications included $\beta$-blockers. No significant differences in response were found between patients who, among other drugs, received $\beta$-blockers and those who did not.

\section{Safety}

No significant differences were found in vital signs, including BP, between insomnia patients with antihypertensive drugs receiving PRM or placebo in the efficacy population at the baseline visit or after 3-week or 6-month PRM treatment (Table 4).

Table 2 Efficacy of PRM vs placebo: improvement in morning alertness compared to placebo (3 weeks)

\begin{tabular}{|c|c|c|c|c|c|c|}
\hline & \multicolumn{3}{|c|}{ PRM } & \multicolumn{3}{|c|}{ Placebo } \\
\hline & $\mathbf{N}$ & Mean (millimeters) & SD & $\mathbf{N}$ & Mean (millimeters) & SD \\
\hline Baseline & 199 & 49.9 & 14.5 & 201 & 50.9 & 14.7 \\
\hline 3 weeks & 195 & 43.1 & 15.1 & 197 & 47.7 & 14.3 \\
\hline Mean change from baseline & 194 & -7.2 & I5.I & 197 & -3.0 & 12.5 \\
\hline Significance PRM vs placebo & \multicolumn{6}{|c|}{$P<0.008$} \\
\hline
\end{tabular}

Abbreviations: PRM, prolonged-release melatonin; SD, standard deviation. 
Table 3 Efficacy of prolonged-release melatonin (PRM) compared with placebo (6 months): improvement in sleep latency (daily sleep diary)

\begin{tabular}{|c|c|c|c|c|c|c|}
\hline \multirow[t]{2}{*}{ Daily sleep diary score } & \multicolumn{3}{|c|}{ PRM } & \multicolumn{3}{|c|}{ Placebo } \\
\hline & $\mathbf{N}$ & $\begin{array}{l}\text { Mean length of } \\
\text { time (minutes) }\end{array}$ & SD & $\mathbf{N}$ & $\begin{array}{l}\text { Mean length of } \\
\text { time (minutes) }\end{array}$ & SD \\
\hline Baseline & 134 & 73.6 & 5.6 & 39 & 73.5 & 4.3 \\
\hline 6 months & 121 & 51.0 & 3.6 & 36 & 65.2 & 4.4 \\
\hline Mean change from baseline & 121 & -23.3 & 2.9 & 36 & -7.5 & 3.6 \\
\hline Significance for PRM vs placebo & \multicolumn{6}{|c|}{$P=0.02$} \\
\hline
\end{tabular}

Abbreviations: PRM, prolonged-release melatonin; SD, standard deviation.

For the AE analysis, altogether, 650 PRM-treated patients and 632 placebo-treated patients with a recorded history of cardiovascular abnormalities or who were diagnosed prior to the trials were evaluated. No changes in vital signs were observed with PRM in this population. The overall rate of AEs recorded in these patients was somewhat higher in PRM (41.8\%) versus placebo-treated patients (36.6\%). However, when normalized per exposure time, the AE occurrences per 100 patient-weeks was much lower for PRM (3.66) than for placebo (8.53). As can be seen in Table 5, the main contributors to the differences in AEs with PRM versus placebo per 100 patient-weeks are gastrointestinal (0.86 vs 1.84$)$, infections and infestations ( 0.95 vs 1.36 ), nervous system disorders ( 0.95 vs 2.66$)$, and psychiatric disorders (0.41 vs 1.81), all of which are lower with PRM than with placebo treatment. Cardiovascular-related AEs were rare $(<0.3 \%)$, with no significant differences between PRM and placebo.

\section{Discussion}

The results of the current post hoc analysis using the primary efficacy endpoints from the individual studies demonstrate that in insomnia patients aged 55 years and older with a history of hypertension and concomitant treatment with antihypertensive drugs, treatment with PRM improves sleep quality and next-day alertness significantly more than placebo. Long-term benefit to these patients was also demonstrated by the significantly greater improvements in sleep latency treated for 6 months with PRM compared with placebo. The effect size of $\sim 0.35$ obtained with PRM in these three sleep variables in comparison with placebo is considered medium, ${ }^{33}$ quite comparable with those of hypnotics, ${ }^{34}$ and well within the range of effect sizes found with central nervous system drugs, ${ }^{35,36}$ and is therefore of clear clinical relevance. Benefit to patients is confirmed by the higher percentage of patients who improved or very much improved in CGI-I with PRM compared with placebo following 6 months of treatment. The safety profile of PRM in this population is benign compared with placebo. This implies that add-on PRM therapy does not present significant risks of detrimental drug interactions with the main drugs used to treat CVD, including hypertension, for long-term periods.

Because hypertension is linked to insomnia, ${ }^{9}$ the efficacy of PRM in improving sleep and morning alertness in patients with insomnia may in part be due to lowering nocturnal BP. Notably, in a 24-hour ambulatory BP monitoring study, $53 \%$ of the patients treated with antihypertensive drugs demonstrated nondipping/nocturnal hypertension despite

Table 4 Blood pressure measurement taken at baseline, 3 weeks, and 6 months as part of safety vital signs collection

\begin{tabular}{|c|c|c|c|c|c|c|c|c|}
\hline & \multicolumn{4}{|l|}{ Baseline } & \multicolumn{4}{|l|}{3 weeks } \\
\hline & \multicolumn{2}{|c|}{$\operatorname{PRM}(n=|3|)$} & \multicolumn{2}{|c|}{ Placebo $(n=185)$} & \multicolumn{2}{|c|}{$\operatorname{PRM}(n=|3|)$} & \multicolumn{2}{|c|}{ Placebo $(n=185)$} \\
\hline & Systolic & Diastolic & Systolic & Diastolic & Systolic & Diastolic & Systolic & Diastolic \\
\hline \multicolumn{9}{|c|}{ Short term } \\
\hline Mean & 132.27 & 76.83 & 132.24 & 76.94 & 131.96 & 75.81 & 130.53 & 75.51 \\
\hline \multirow[t]{4}{*}{ SD } & $15.9 \mid$ & 8.09 & 14.4 & 8.56 & 13.76 & 7.68 & 13.53 & 8.86 \\
\hline & \multicolumn{4}{|l|}{ Baseline } & \multicolumn{4}{|c|}{6 months } \\
\hline & \multicolumn{2}{|c|}{$\operatorname{PRM}(n=125)$} & \multicolumn{2}{|c|}{ Placebo $(n=25)$} & \multicolumn{2}{|c|}{$\operatorname{PRM}(n=125)$} & \multicolumn{2}{|c|}{ Placebo $(n=25)$} \\
\hline & Systolic & Diastolic & Systolic & Diastolic & Systolic & Diastolic & Systolic & Diastolic \\
\hline \multicolumn{9}{|c|}{ Long term } \\
\hline Mean & 131.25 & 76.36 & 125.69 & 75.23 & 128.73 & 73.72 & 124.13 & 75.29 \\
\hline SD & 16.3 & 8.7 & 12.5 & 8.2 & 15.8 & 7.2 & 10.3 & 6.0 \\
\hline
\end{tabular}

Abbreviations: PRM, prolonged-release melatonin; SD, standard deviation. 


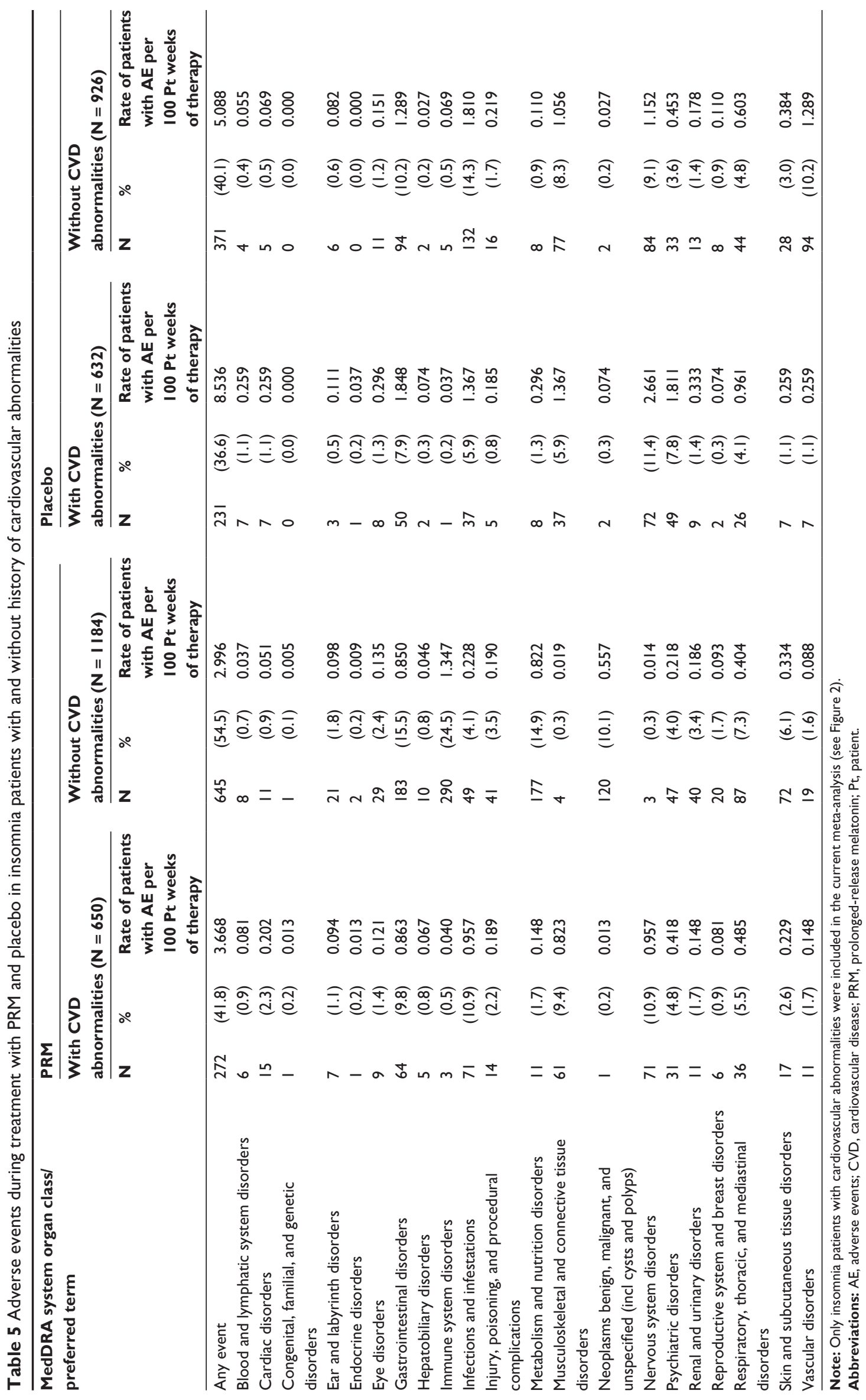


pharmacotherapy, ${ }^{37}$ suggesting that antihypertensive treatment does not restore proper circadian rhythms in BP. PRM and other controlled-release melatonin formulations $(2-3 \mathrm{mg})$ but not immediate-release formulations have been consistently shown to reduce nocturnal BP. ${ }^{16}$ The other way around is less likely, as improvement in sleep alone, such as with benzodiazepine/benzodiazepine-like hypnotics, does not improve nocturnal hypertension. In fact, zolpidem, the most commonly used hypnotic drug, does not lessen, and can even increase, nocturnal BP. ${ }^{38,39}$

A limitation in our study is that no measurements of nocturnal BP were taken in the clinical trials included in the combined analysis. However, it is important to note that BP (daytime) measured in all of the studies as part of general safety vital sign assessments was not impaired with PRM compared with placebo, suggesting that PRM did not reduce the efficacy of the antihypertensive therapy. These data agree well with studies on the effects of PRM and controlledrelease melatonin preparations in patients with nocturnal hypertension, all of which showed a decrease in BP during the night and no change during the day (reviewed by Grossman et al). ${ }^{16}$ Therefore, it is possible that the improvement in nocturnal hypertension augments the soporific effects of PRM in insomnia patients who are treated with antihypertensive drugs. Both effects may nevertheless be related to the effects of PRM on the biological clock regulating the day-night cycles in sleep and wakefulness and BP. An ambulatory BP monitoring study examining the effect of PRM on insomnia patients with hypertension to shed more light on the inter-relation between insomnia and nocturnal $\mathrm{BP}$ is thus warranted.

All of the patients in the hypertensive insomnia subpopulation in our studies were treated with antihypertensive medications (mostly ß-blockers, calcium channel blockers, and angiotensin-converting enzyme inhibitors). Nevertheless, because most of the patients received two and sometimes three or four antihypertensive medications, we could not determine whether the improvement in sleep and daytime parameters with PRM was specifically associated with the concomitant use of a particular antihypertensive drug. Specifically, $ß$-blockers are known to reduce endogenous melatonin secretion ${ }^{40}$ and induce insomnia. ${ }^{41}$ We did not observe major differences in response to PRM between patients treated with $\beta$-blockers or not treated with $\beta$-blockers. This is perhaps because melatonin is low in patients with hypertension or coronary heart disease, ${ }^{42-45}$ or because some of the $\beta$-blockers used do not affect melatonin. ${ }^{46,47}$ Altogether, PRM appears to be effective and safe for the treatment of insomnia in patients with insomnia and cardiovascular comorbidity, including hypertension, and may therefore serve as the first choice of hypnotics for patients with hypertension.

\section{Acknowledgment/disclosure}

All the studies described in this article were supported by Neurim Pharmaceuticals (1991) Ltd, the manufacturer of PRM $\left(\right.$ Circadin $\left.^{\circledR}\right)$. The authors report no other conflicts of interest in this work.

\section{References}

1. Bliwise DL, King AC, Harris RB, Haskell WL. Prevalence of selfreported poor sleep in a healthy population aged 50-65. Soc Sci Med. 1992;34(1):49-55.

2. Fullerton DS. The economic impact of insomnia in managed care: a clearer picture emerges. Am J Manag Care. 2006;12(Suppl 8):S246-S252.

3. Leger D, Poursain B, Neubauer D, Uchiyama M. An international survey of sleeping problems in the general population. Curr Med Res Opin. 2008;24(1):307-317.

4. Ohayon MM, Sagales T. Prevalence of insomnia and sleep characteristics in the general population of Spain. Sleep Med. 2010;11(10):1010-1018.

5. Roth T. Insomnia: definition, prevalence, etiology, and consequences. J Clin Sleep Med. 2007;3(Suppl 5):S7-S10.

6. Roth T, Roehrs T. Insomnia: epidemiology, characteristics, and consequences. Clinical Cornerstone Chronic Insomnia. 2003; 5(3):5-15.

7. Egan BM, Zhao Y, Axon RN. US trends in prevalence, awareness, treatment, and control of hypertension,1988-2008. JAMA. 2010; 303(20):2043-2050.

8. Wolf-Maier K, Cooper RS, Banegas JR, et al. Hypertension prevalence and blood pressure levels in 6 European countries, Canada, and the United States. JAMA. 2003;289(18):2363-2369.

9. Roth T. Comorbid insomnia: current directions and future challenges. Am J Manag Care. 2009;Suppl 15:S6-S13.

10. Lanfranchi PA, Pennestri MH, Fradette L, Dumont M, Morin CM, Montplaisir J. Nighttime blood pressure in normotensive subjects with chronic insomnia: implications for cardiovascular risk. Sleep. 2009;32(6):760-766

11. Gangwisch JE, Malaspina D, Posner K, et al. Insomnia and sleep duration as mediators of the relationship between depression and hypertension incidence. Am J Hypertens. 2010;23(1):62-69.

12. Gangwisch JE, Heymsfield SB, Boden-Albala B, et al. Short sleep duration as a risk factor for hypertension: analyses of the first National Health and Nutrition Examination Survey. Hypertension. May 2006; 47(5):833-839.

13. Fung MM, Peters K, Redline S, et al. Decreased slow wave sleep increases risk of developing hypertension in elderly men. Hypertension. 2011;58(4):596-603.

14. Hermida RC, Ayala DE, Mojon A, Fernandez JR. Decreasing sleep-time blood pressure determined by ambulatory monitoring reduces cardiovascular risk. J Am Coll Cardiol. 2011;58(11):1165-1173.

15. Hoevenaar-Blom MP, Spijkerman AMW, Kromhout D, van den Berg JF, Verschuren WMM. Sleep duration and sleep quality in relation to 12-year cardiovascular disease incidence: the MORGEN Study. Sleep. In press. 2011.

16. Grossman E, Laudon M, Zisapel N. Effect of melatonin on nocturnal blood pressure: meta-analysis of randomized controlled trials. Vasc Health Risk Manag. 2011;7:577-584.

17. Wade A, Zisapel N, Lemoine P. Prolonged-release melatonin for the treatment of insomnia: targeting quality of sleep and morning alertness. Aging Health. 2008;1:11-21

18. Czeisler CA, Duffy JF, Shanahan TL, et al. Stability, precision, and near-24-hour period of the human circadian pacemaker. Science. 1999;284(5423):2177-2181. 
19. Hofman MA, Swaab DF. Alterations in circadian rhythmicity of the vasopressin-producing neurons of the human suprachiasmatic nucleus (SCN) with aging. Brain Res. 1994;651(1-2):134-142.

20. Mahlberg R, Kienast T, Hadel S, Heidenreich JO, Schmitz S, Kunz D. Degree of pineal calcification (DOC) is associated with polysomnographic sleep measures in primary insomnia patients. Sleep Med. 2009;10(4):439-445.

21. Waldhauser F, Weiszenbacher G, Tatzer E, et al. Alternations in nocturnal serum melatonin levels in humans with growth and aging. J Clin Endocrinol Metab. 1988;66:648-652.

22. Haimov I, Laudon M, Zisapel N, et al. Sleep disorders and melatonin rhythms in elderly people. BMJ. 1994;309:167.

23. Leger D, Laudon M, Zisapel N. Nocturnal 6-sulfatoxymelatonin excretion in insomnia and its relation to the response to melatonin replacement therapy. Am J Med. 2004;116(2):91-95.

24. Lemoine P, Garfinkel D, Laudon M, Nir T, Zisapel N. Prolonged release melatonin for insomnia - an open label long term study of efficacy, safety and withdrawal symptoms Ther Clin Risk Manag. 2011;7: 301-311.

25. Lemoine P, Nir T, Laudon M, Zisapel N. Prolonged-release melatonin improves sleep quality and morning alertness in insomnia patients aged 55 years and older and has no withdrawal effects. J Sleep Res. 2007; $16: 372-380$.

26. Luthringer R, Muzet M, Zisapel N, Staner L. The effect of prolongedrelease melatonin on sleep measures and psychomotor performance in elderly patients with insomnia. Int Clin Psychopharmacol. 2009;24(5): 239-249.

27. Wade AG, Ford I, Crawford G, et al. Nightly treatment of primary insomnia with prolonged release melatonin for 6 months: a randomized placebo controlled trial on age and endogenous melatonin as predictors of efficacy and safety. BMC Med. 2010;8(1):51.

28. Wade AG, Ford I, Crawford G, et al. Efficacy of prolonged release melatonin in insomnia patients aged 55-80 years: quality of sleep and next-day alertness outcomes. Curr Med Res Opin. 2007;23(10): 2597-2605.

29. Zisapel N. Laudon M. Subjective assessment of the effects of CNSactive drugs on sleep by the Leeds Sleep Evaluation Questionnaire: a review. Human Psychopharmacology Clin Exp. 2002;17:1-19.

30. Parrott AC, Hindmarch I. The Leeds Sleep Evaluation Questionnaire in psychopharmacological investigations - a review. Psychopharmacology (Berl). 1980;71(2):173-179.

31. Tarrasch R, Laudon M, Zisapel N. Cross-cultural validation of the Leeds Sleep Evaluation Questionnaire (LSEQ) in insomnia patients. Hum Psychopharmacol. 2003;18(8):603-610.

32. Guy W. ECDEU assessment manual for psychopharmacology (rev, 1976): Kensington: DHEW publication. Biometric laboratory, George Washington University, US Department of Health, Education and Welfare; 1976.
33. Cohen J. Statistical power analysis for the behavioral sciences. Hillsdale, NJ: Lawrence Earlbaum Associates; 1988.

34. Glass J, Lanctot KL, Herrmann N, Sproule BA, Busto UE. Sedative hypnotics in older people with insomnia: meta-analysis of risks and benefits. BMJ. 2005;331(7526):1169.

35. Claghorn JL, Kiev A, Rickels K, Smith WT, Dunbar GC. Paroxetine versus placebo: a double-blind comparison in depressed patients. J Clin Psychiatry. 1992;53(12):434-438.

36. Schneider LS, Ismail MS, Dagerman K, et al. Clinical Antipsychotic Trials of Intervention Effectiveness (CATIE): Alzheimer's disease trial. Schizophr Bull. 2003;29(1):57-72.

37. de la Sierra A, Redon J, Banegas JR, et al. Prevalence and factors associated with circadian blood pressure patterns in hypertensive patients. Hypertension. 2009;53:466-472.

38. McCann CC, Quera-Salva MA, Boudet J, et al. Effect of zolpidem during sleep on ventilation and cardiovascular variables in normal subjects. Fundam Clin Pharmacol. 1993;7(6):305-310.

39. Rachmani R, Shenhav G, Slavachevsky I, Levy Z, Ravid M. Use of a mild sedative helps to identify true non-dippers by ABPM: a study in patients with diabetes mellitus and hypertension. Blood Press Monit. 2004;9(2):65-69.

40. Rommel T, Demisch L. Influence of chronic b-adrenoreceptor blocker treatment on melatonin secretion and sleep quality in patients with essential hypertension. J Neural Transm. 1994;95:39-48.

41. Tanabe N, Fujita T, Fujii Y, Orii T. Investigation of the factors that contribute to the onset of insomnia in hypertensive patients by using a post-marketing surveillance database. Yakugaku Zasshi. 2011; 131(5):669-677. Japanese.

42. Brugger P, Marktl W, Herold M. Impaired nocturnal secretion of melatonin in coronary heart disease. Lancet. 1995;345(8962):1408.

43. Jonas M, Garfinkel D, Zisapel N, Laudon M, Grossman E. Impaired nocturnal melatonin secretion in non-dipper hypertensive patients. Blood Press. 2003;12:19-24.

44. Rapoport SI, Shatalova AM, Malinovskaia NK, Vettenberg L. Melatonin production in hypertensive patients. Klin Med (Mosk). 2000;78(6): 21-24. Russian.

45. Sakotnik A, Liebmann PM, Stoschitzky K, et al. Decreased melatonin synthesis in patients with coronary artery disease. Eur Heart J. 1999;20(18):1314-1317.

46. Stoschitzky K, Sakotnik A, Lercher P, et al. Influence of beta-blockers on melatonin release. Eur J Clin Pharmacol. 1999;55(2):111-115.

47. Stoschitzky K, Stoschitzky G, Brussee H, Bonelli C, Dobnig H Comparing beta-blocking effects of bisoprolol, carvedilol and nebivolol. Cardiology. 2006;106(4):199-206.
Integrated Blood Pressure Control

\section{Publish your work in this journal}

Integrated Blood Pressure Control is an international, peer-reviewed open-access journal focusing on the integrated approach to managing hypertension and risk reduction. Treating the patient and comorbidities together with diet and lifestyle modification and optimizing healthcare resources through a multidisciplinary team approach constitute key

\section{Dovepress}

features of the journal. This journal is indexed on American Chemical Society's Chemical Abstracts Service (CAS). The manuscript management system is completely online and includes a very quick and fair peerreview system, which is all easy to use. Visit http://www.dovepress.com/ testimonials.php to read real quotes from published authors. 